\title{
Rabies virus glycoprotein enhances spatial memory via the PDZ binding motif
}

Soheil Ghassemi ${ }^{1}$, Tara Asgari ${ }^{1}$, Hamid Gholami Pourbadie ${ }^{1 *}$, Christophe Prehaud ${ }^{2}$, Monique Lafon ${ }^{2}$, Nima Naderi ${ }^{3}$, Alireza Gholami ${ }^{4}$, Kayhan Azadmanesh ${ }^{5}$, Mohammad Sayyah $^{1 *}$

1- Department of Physiology and Pharmacology, Pasteur Institute of Iran, Tehran, Iran

2- Institut Pasteur, Unité de Neuroimmunologie Virale, UMR 3569, CNRS, Paris, France

3- Department of Pharmacology and Toxicology, School of Pharmacy, Shahid Beheshti University of Medical Sciences, Tehran, Iran

4- WHO Collaborating Center for Reference and Research on Rabies, Pasteur Institute of Iran, Tehran, Iran

5- Department of Virology, Pasteur Institute of Iran, Tehran, Iran.

\section{Corresponding authors:}

\section{1- Mohammad Sayyah}

Department of Physiology and Pharmacology

Pasteur Institute of Iran, Tehran, Iran

Telephone: +98 2164112834

E-mail: sayyahm2@pasteur.ac.ir

\section{2- Hamid Gholami Pourbadie}

Department of Physiology and Pharmacology

Pasteur Institute of Iran, Tehran, Iran

Telephone: +98 2164112834

E-mail: h_gholamipour@pasteur.ac.ir 


\begin{abstract}
Rabies is a life-threatening viral infection of the brain. Rabies virus (RABV) merely infects excitable cells including neurons provoking drastic behaviors including negative emotional memories. RABV glycoprotein (RVG) plays a critical role in RABV pathogenesis. RVG interacts with various cytoplasmic PDZ (PSD-95/Dlg/ZO-1) containing proteins through its PDZ binding motif (PBM). PTZ domains have crucial role in formation and function of signal transduction. Hippocampus is one of the cerebral regions that contains high load of viral antigens. We examined impact of RVG expression in the dorsal hippocampus on aversive as well as spatial learning and memory performance in rats.
\end{abstract}

Two $\mu$ l of the lentiviral vector $\left(\sim 10^{8}\right.$ T.U. /ml) encoding RVG or $\Delta \mathrm{RVG}$ (deleted PBM) genomes was microinjected into the hippocampal CA1. After one week, rat's brain was cross-sectioned and RVG/ARVG-expressing neuronal cells were confirmed by fluorescent microscopy. Passive avoidance and spatial learning and memory were assessed in rats by Shuttle box and Morris water maze (MWM).

In the shuttle box, both RVG and $\triangle \mathrm{RVG}$ decreased the time spent in the dark compartment compared to control $(p<0.05)$. In MWM, RVG and $\triangle \mathrm{RVG}$ did not affect the acquisition of spatial task. RVG-expressing rats reached the platform position in the probe test sooner than control and $\triangle \mathrm{RVG}$ groups $(p<0.05)$. Rats expressing $\triangle \mathrm{RVG}$ significantly swam farther from the hidden platform than RVG group $(p<0.05)$.

Our data indicate RVG expression in the hippocampus strengthens aversive and spatial learning and memory performance. The boosting effect on spatial but not avoidance memory is mediated through PBM.

Keywords: rabies glycoprotein, learning and memory, CA1, PDZ binding motif, dorsal hippocampus

\title{
1. Introduction
}

Rabies is an infectious disease with neurologic manifestations, which is caused by a neurotropic virus from family Rhabdoviridae, genus Lyssavirus, and subgroup Rabies virus (RABV). The virus infects all mammals, including humans. After inoculation, virions transport in retrograde 
direction along axons and across synapses to the central nervous system (CNS). RABV infects neuronal cells and provokes drastic behavior perturbation including anxiety, rage, hallucinations, depression, and aero-/hydro-phobia. However, the corresponding molecular mechanism(s) is still undiscovered. Relative slow replication rate of pathogenic strains provides viral dissemination throughout the brain with minor stimulation of cerebral immune system and lack of prominent neurodegeneration. Despite drastic affective disorders such as anxiety, rage, hallucinations, depression, and ultimately death, only minor histological changes are seen in CNS of rabies patients. This fact raises the possibility that not the neurostructural and neurodegenerative changes rather neuronal dysfunctions underlie the clinical manifestation and lethality of RABV (Jackson, 2016). This suggestion is supported by numerous reports indicating modulation of synaptic transmission by RABV including ion channel dysfunction (Iwata et al, 1999), aberrant neurotransmission (Ceccaldi et al, 1993; Dumrongphol et al, 1996; Ladogana et al, 1994), and changes in the electroencephalography of rabies patients (Gourmelon et al, 1991). Furthermore, in the previous study we showed that lentiviral expression of the rabies glycoprotein enhances synaptic transmission in the hippocampal dentate gyrus region (Ghassemi et al, 2021). Another clue that strengthens changes in synaptic function by RABV is that RABV increases release of neurotransmitters including serotonin and catecholamines in the hippocampus of the infected mice for a period of 1 week after infection with a peak on the day 3 (Fu and Jackson, 2005). Meanwhile, microstructural changes in neuronal processes and synapses including disorganization of apical dendrites are observed in the hippocampus of mice infected with the pathogenic $\mathrm{N} 2 \mathrm{C}$ strain of RABV (Préhaud et al, 2010). Yet, minimal TUNEL positive inflammatory cells confirming no apparent neuronal death are detected in the cerebral cortex and hippocampus of moribund YFP mice peripherally infected with challenge virus standard (CVS) (Scott et al, 2008). Therefore, it seems that the behavioral changes seen during the early stages of the infection before death, in part, is due to the increase in synaptic or neuronal activity by RABV.

RABV encodes five structural proteins including glycoprotein, phosphoprotein, nucleoprotein, matrix protein and RNA-dependent RNA polymerase. Among these proteins, only RABV glycoprotein (RVG) is inserted into the host cell membrane. It is a single pass membrane protein with extracellular $\mathrm{N}$-terminus and cytoplasmic $\mathrm{C}$-terminus that accounts for RABV binding to the host cell membrane. C-terminal of the RVG has been reported to interact with PDZ containing mediators within the host cell. The PDZ domain is a common structural domain of 80-90 amino- 
acids found in more than 150 proteins. PDZ domains bind to a short region of the C-terminus of other specific proteins. These domains play a key role in the formation and function of signal transduction complexes (Nourry et al, 2003). Having different sequences at the C-terminals, diverse strains of RABV induce divergent response of the host cell. In this regard, it has been reported that the virulent form of RABV promotes cell preservation and survival through its $\mathrm{C}$ terminal interaction with the PDZ domain of microtubule-associated serine and threonine kinase 2 (MAST2) inside the host cell, and therefore boosts viral dissemination (Caillet-Saguy et al, 2015). On the other hand, nonpathogenic strains trigger neuronal apoptosis through involving different host cell mediator (Lafon, 2011; Prehaud et al, 2003). The role of RVG in neuronal protection as well as its potential impacts on synaptic function strengths the possibility that RVG plays a role in the behavior of rabies patient. In this regard, Khan et al., have recently shown an accelerated neurite outgrowth in different neuronal cell lines expressing RVG-derived isoforms (Khan et al, 2019) suggesting a neuroprotective role of RVG. In addition, Hueffer et al., reported that a RVG-derived peptide modifies motor behavior through inhibition of nicotinic acetylcholine receptors (Hueffer et al, 2017).

Learning and memory are essential brain functions that allow the individual to adapt to harmful or pleasant experiences by formation of new neural networks in the brain. The cellular basis of learning and memory is the strengthening or weakening of synaptic contacts as a result of increasing or decreasing activity levels of the neurons involved in a particular neural circuit. Hippocampus is a brain structure embedded deep in the temporal lobe of each cerebral cortex. It is an important part of the limbic system, a cortical region that regulates motivation, emotion,

learning, and memory. This curled structure is highly infected by RABV. Given the strong evidence regarding modification of synaptic function by RVG, and emotional changes in rabies patients, we aimed to explore the effect of RVG on the two main type of learning and memory tasks i.e., spatial and aversive memories in rats.

\section{Materials and methods}

\subsection{Lentiviral vector production}

The lentiviral vectors were made according to the method, which is described previously (Bernier et al, 2017). Briefly, HEK 293T cells were cultured in Dulbecco's Modified Eagle Medium (DMEM, with $10 \% \mathrm{FBS}$ and $100 \mathrm{units} / \mathrm{ml}$ penicillin/streptomycin) at $37^{\circ} \mathrm{C}$ in a humidified $5 \%$ 
$\mathrm{CO} 2$ incubator. Plasmids encoding full-length RVG of challenge virus standard CVS (G-CVS) or $\triangle \mathrm{RVG}$, with four amino acids deletion in the PBM site (Fig. 1) (Khan et al, 2019), were kindly provided by Christophe Prehaud (Neuroimmunologie Virale, Institute Pasteur, Paris, France) and sub-cloned in Plenti7.3 with eGFP genome (Invitrogen, France) containing cytomegalovirus (CMV) promoter. The HEK 293T cells were transfected with envelope ( $\mathrm{G}$ protein of VSV), transfer (p-Lenti-G-CVS) and packaging (pMDLg, pRRE and pRSV/Rev) plasmids by calcium phosphate method. Twenty four h later, the medium was replaced by fresh medium containing $2 \%$ FBS and the cells were incubated for two days. The medium was then collected and centrifuged at $1000 \mathrm{~g}, 4^{\circ} \mathrm{C}$ for five min and the supernatant was filtered through a $0.45-\mu \mathrm{m}$ poly ether sulfone filter. Viral particles were centrifuged at $50000 \times \mathrm{g}$ at $4^{\circ} \mathrm{C}$ for $2 \mathrm{~h}$. The pellet was suspended in phosphate-buffered saline (PBS)-BSA 1\% solution. Different aliquots were then prepared and kept in $-80^{\circ} \mathrm{C}$ until use.

\subsection{Determination of viral titration}

HEK $293 \mathrm{~T}$ cells were cultured in a 24-well plate containing $0.5 \mathrm{ml}$ medium with 5\% FBS and incubated at $37^{\circ} \mathrm{C}$ with $5 \% \mathrm{CO} 2$ overnight. Then, they were transduced by the serial dilutions of the lentiviral vector. GFP-positive cell number on each dilution was determined under a fluorescent microscope (Nikon, Japan) at the day 3. The functional vector titer (transducing units, TU) was measured using the following formula:

Titer $(\mathrm{TU} / \mathrm{ml})=[$ GFP-positive cells $\times$ number of cells on the transduction day $(5 \times$ $\left.\left.10^{5}\right)\right] /$ dilution

\subsection{Animals and Surgical procedures}

Adult male Wistar rats (220-250g, Pasteur Institute of Iran, $\mathrm{n}=60$ ) were used in this study. They were housed in groups of four in clear cages with free access to food and water in a $12 \mathrm{~h}$ light/12hdark cycle. All efforts were made to use minimal number of rats and decrease animal suffering during the experiments. Experiments were performed according to the Review Board and Ethics Committee of Pasteur Institute of Iran (Authorization code 93-0201-785, 22 April 2014) and conform to the European Communities Council Directive of 24 November 1986 (86/609/EEC). Animals were allocated to 3 groups as RVG $(n=20), \Delta R V G(n=16)$, and control $(n=24)$. Control group was divided into two subgroups as naïve intact $(\mathrm{n}=6)$ and phosphate-buffered saline (PBS, 
$\mathrm{n}=18)$. Rats were anesthetized by intraperitoneal injection of ketamine (100 mg/kg) and xylazine $(10 \mathrm{mg} / \mathrm{kg})$. The procedure of injection into hippocampal CA1 area was performed by stereotaxic surgery according to the previously described method (Farzaneh et al., 2018). Briefly, $2 \mu 1$ of RVG, or $\triangle \mathrm{RVG}$ lentivirus, or PBS, were delivered into $\mathrm{CA} 1$ area $(\mathrm{AP}=-3.6, \mathrm{ML}= \pm 2, \mathrm{DV}=-2.2)$ through a Hamilton microsyringe connected to microinfusion pump, according to coordinates of the rat brain atlas (Paxinos and Watson, 2006).

\subsection{Histology \& Immunohistochemistry}

One week after microinjection of the lentivector into the CA1, brain of the rats ( 3 rats in each group) was cut up for histological examinations. The animals were anesthetized and perfused transcardially with cold solution of $4 \%$ paraformaldehyde dissolved in $0.1 \mathrm{M}$ PBS. Brains were then removed, post-fixed in $2 \%$ paraformaldehyde for two days, processed and embedded in paraffin blocks. Using a microtome, the brains were horizontally sliced into $5 \mu \mathrm{m}$ thickness. The sections were mounted with $90 \%$ glycerol/PBS buffer and observed in the dark room under fluorescent microscope (Nikon, Japan) equipped with specific filter cube for FITC fluorescence channels. Digital photographs were taken using $4 x$ and 20x objective lenses.

In order to assess any histopathological changes in CA1 area, the brain slices were rehydrated, and stained with Hematoxylin \& Eosin (Sigma-Aldrich, Germany).

To detect any neuroinflammatory changes in CA1, the gliosis in areas with GFP-positive cells was assessed by immunostaining for GFAP (glial fibrillary acidic protein) as the specific marker of microglia. After rehydration of the sections, they were rinsed three times (10 min each) by PBS and incubated overnight at $4{ }^{\circ} \mathrm{C}$ in a dark place with monoclonal anti-glial fibrillary acidic protein (anti-GFAP) anti-body (Merck Millipore, dilution 1:400). The sections were then incubated with Texas Red-X conjugated goat anti-mouse IgG secondary antibody (Merck Millipore, dilution 1:1000) for $3 \mathrm{~h}$. Then sections were washed for 5 min with PBS and cover slipped with $90 \%$ glycerol mounting buffer and visualized in a dark place with a fluorescence microscope (Nikon), equipped with specific filter cube for fluorescein Texas Red-X and FITC fluorescence channels, and connected to a digital camera. Digital photographs were taken using 4 and $20 \times$ objective lenses.

\subsection{Spatial learning and memory}


Spatial learning and memory of animals was assessed according to the previously described method (Pourbadie et al, 2018). The water maze consisted of a pool (155 cm diameter) filled with tap water $\left(22 \pm 2^{\circ} \mathrm{C}\right)$ located in a room with walls decorated with distinct extra maze (spatial) cues. A hidden Plexiglas platform (10 cm diameter) was positioned $1.5 \mathrm{~cm}$ below the water surface in the target quadrant. A CCD camera (Panasonic Inc, Japan) hanging from the ceiling above the tank recorded animal's locomotion, then a video tracking software (Ethovision, versionXT7, The Netherlands) digitized and saved the locomotion to the computer. The animals were trained to find a fixed hidden platform submerged in the target quadrant during 60 seconds while they were released into the tank from different directions. The rats that could not find the platform within 60 seconds were directed toward platform and were allowed to rest on it for 10 seconds. Animals performed one training session of four trials for three consecutive days. Twenty four $h$ after the last training session probe test was performed so that the platform was removed and the rats were allowed to swim 60 seconds. After the probe test, rats were evaluated to find a visible platform that was elevated from water surface and covered with a bright aluminum foil in order to test their visual ability. The parameters of escape latency, traveled distance, time spent in target quadrant, and swim velocity were recorded for subsequent analysis.

\subsection{Passive avoidance learning and memory}

The shuttle box apparatus contains two chambers, bright and dark $(20 \mathrm{~cm} \times 20 \mathrm{~cm} \times 30 \mathrm{~cm})$, separated by a guillotine door $(7 \mathrm{~cm} \times 9 \mathrm{~cm})$. Bottom of the chambers are made of stainless steel grids $(2.5 \mathrm{~mm}$ diameter) with $1 \mathrm{~cm}$ intervals. The bottom of the dark compartment is connected to an insulated stimulator. At the first day, the animal was placed in the bright chamber and guillotine door was opened allowing him to explore the apparatus for $60 \mathrm{sec}$. Thirty min later, the procedure was repeated and a foot shock $(50 \mathrm{~Hz}, 1.5 \mathrm{~mA}$ for $1.5 \mathrm{sec})$ was applied once the animal entered the dark chamber (Kheirbek et al, 2013). Twenty seconds later, the animal was removed and returned to its cage. Two min later, the procedure was repeated and the acquisition was attained if the rat would not enter the dark chamber over $120 \mathrm{sec}$. Twenty h later, the rat was placed in the light chamber, the door was opened and its behavior was monitored for $10 \mathrm{~min}$. The parameters of initial latency, step-through latency (STL) and the time spent in the dark compartment (TDC) were recorded.

\subsection{Statistical analysis}


The data are expressed as means \pm S.E.M and processed by commercially available software Graph Pad Prism 6.0. One-way analysis of variance (ANOVA) and post-hoc Tukey's test were used for multiple comparisons. The escape latency and distance to platform in the training day were analyzed using two way ANOVA and Bonferroni post- hoc test. In all cases, a $p \leq 0.05$ was considered statistically significant.

\section{Results}

\subsection{RVG was efficiently expressed in $C A 1$}

A $10^{8} \mathrm{TU} / \mathrm{ml}$ titer was found for the vector. Fig. 2 shows representative fluorescent images from control and RVG groups. RVG/eGFP gene was successfully expressed in pyramidal neurons one week after microinjection of the lentivector into the CA1 (Fig. 2). Over $90 \%$ of the CA1 pyramidal cells in the injection site expressed bright green fluorescence at the day 7.

\subsection{Lentiviral particles did not induce neuroinflammation or neuron loss in the injection site.}

As depicted in Fig. 3A, no obvious morphological changes or cell loss was detected in the injection site of lenitvirus vector or PBS. Furthermore, no significant difference was found between PBS and lentiviral vector in number and morphology of GFAP positive cells in the injection site (Fig. 3B).

\subsection{RVG boosts spatial learning and memory performance}

No significant difference was found between naïve control and PBS groups. Therefore, the data of naïve control is not presented. Reference spatial learning and memory was evaluated one week after delivery of RVG or $\triangle \mathrm{RVG}$ into the CA1. Two-way ANOVA revealed significant difference of the time factor $[\mathrm{F}(2,75)=27.46, p<0.0001]$ but not the treatment factor or the interaction. Fig. 4A shows no significant difference between groups indicating that RVG or $\triangle \mathrm{RVG}$ did not affect the acquisition of spatial task. Moreover, no significant difference in the distance swimming from the hidden platform was observed among groups during training days (Fig. 4B).

Fig. 5A represents swimming path in the tank during the probe test. In the probe test, as seen in Fig. 5B, one-way ANOVA $[\mathrm{F}(2,24)=4.312, p=0.025]$ followed by Tukey's post-test revealed that expression of RVG in the CA1 $(n=11)$ increases swimming time in the target quadrant compared to the PBS group ( $\mathrm{p}=0.019, \mathrm{n}=9$ ). However, no difference was observed between 
$\triangle \mathrm{RVG}(\mathrm{n}=6)$ and PBS groups. Fig. $4 \mathrm{C}$ shows a significant difference of mean distance swimming from the hidden platform between RVG and PBS group $(p=0.041)$. Rats expressing $\Delta \mathrm{RVG}$ significantly swam farther from the hidden platform than RVG group $(p<0.036)$. There is no significant difference between PBS and $\triangle \mathrm{RVG}$ groups. RVG-treated group also reached the platform position in the probe test sooner than PBS group (Fig. 5D, $p<0.024$ ).

Fig. 6 shows that there was no difference of velocity (A) or distance moved in the probe test among groups. In addition, no difference of escape latency in the visible test was observed among groups indicating $\mathrm{RVG}$ or $\triangle \mathrm{RVG}$ did not affect visuomotor capability of rats.

\subsection{RVG enhances passive avoidance memory}

Passive avoidance memory was assessed one week after delivery of RVG lentivector into the CA1. The initial latency was not significant among the groups (Fig. 7A) indicating no effect of RVG on visuomotor activity. One way ANOVA followed by Tukey's multiple comparisons test revealed a significant difference among groups $[\mathrm{F}(2,16)=4.482 ; p=0.0285]$. Compared to PBS $(\mathrm{n}=6)$, both RVG $(n=7)$ and $\triangle \mathrm{RVG}(\mathrm{n}=6)$ groups showed greater latency to enter the dark compartment (Fig. 7B). There is a significant difference between PBS and RVG or $\Delta \mathrm{RVG}$ groups $(p<0.05)$. Fig. 7C shows that both RVG and $\triangle \mathrm{RVG}$ decreased the time spent in the dark compartment. Oneway ANOVA showed significant difference of the time spent in dark compartment between groups $[\mathrm{F}(2,14)=3.531 ; p<0.05]$. Tukey's post hoc test showed significant difference between PBS and RVG or $\Delta \mathrm{RVG}$ groups $(p<0.05)$.

\section{Discussion}

The main finding of this study is that rabies glycoprotein (RVG) boosts learning and memory performance. We found that expressing RVG in the dorsal hippocampus facilitates spatial learning and memory through a PBM-dependent manner. Both RVG and $\triangle \mathrm{RVG}$ facilitated passive avoidance memory. Therefore, it could not be firmly concluded that RVG reinforces aversive memory.

In our study, we selected the 1-week period after injection of the virus for evaluation of memory performance. This time period was selected based on the preliminary studies performed in our lab. At this time period, near to $90 \%$ of the pyramidal neurons of the vector-injected hippocampal area expressed GFP. We previously reported frequency of GFP-expressing cells in the CA1 during a 60 days period after injection of a lentiviral vector with the same construct 
(except than the inserted gene) as used in the present study. We found that more than $90 \%$ of the counted CA1 pyramidal neurons are infected one week after injection of the vector into this area. This transduction efficiency reached $95 \%$ two weeks after injection of the virus and preserved for 60 days (Farzaneh et al, 2018). In addition, since behavioral changes appear rapidly after rabies infection, we intended to know the effect of rabies glycoprotein on the memory at the early times after infection of the hippocampus. Therefore, we examined impact of rabies glycoprotein on memory 1 week after infection of the hippocampus.

Excessive sensitivity to water, light, sound, wind and pain all indicate overactivity of the pertinent neural networks in the brain of rabies patients. In this regard, the street strain of rabies virus has been reported to disappear all stages of sleep waves in mice and increases duration of wakefulness (Gourmelon et al, 1991) suggesting hyper-excitability of the neurons involved in sleep-wake cycle. These findings indicate that rabies virus induces drastic neurological manifestations through deregulating network function and synaptic transmissions in the brain. In this line, it has been reported that synaptic transmission of serotonin (Bouzamondo et al, 1993), acetylcholine (Dumrongphol et al, 1996), GABA (Ladogana et al, 1994) and dopamine (Fu and Jackson, 2005) are changed in the brain infected by rabies virus. The altered synaptic transmission can be provoked by proteins encoded by the rabies virus. In agreement with this proposal, a recent study showed that a peptide derived from RVG, which contains a snake toxin-like sequence, changes synaptic transmissions and motor behavior through blocking acetylcholine receptors (Hueffer et al, 2017). In addition, we recently showed that RVG strengthens both short- and longterm synaptic plasticity in the hippocampal dentate gyrus suggesting that it may alter synaptic function through both pre- and postsynaptic mechanisms (Ghassemi et al, 2021). In line with theses evidences, we found in the present study that RVG expression in the dorsal hippocampus facilitates spatial learning and memory. Despite drastic behavioral alterations, histopathological changes are surprisingly mild in the brain of patient infected with rabies virus. Yet, in order to rule out any potential neuropathologic effect of RVG hippocampal expression on the learning and memory performance of rats, we examined a very detailed view of the tissue by H\& E staining as well as immunostaining of the astrocytes at the site of RVG lentivector delivery, CA1. Astrocytes are a sub-type of glial cells in CNS, which react to CNS challenges by hypertrophy, proliferation, and overexpression of their GFAP near the site of injury. The increased GFAP immunoreactivity is the crucial index of gliosis and correlates with neural damage. We did not find any change in structure 
of neuronal cells and also astrocyte morphology and/or overexpression of GFAP in CA1 of the infected rats.

Brain viral loads of rabies virus are particularly high in the hippocampus (Song et al, 2013). Since the hippocampus plays a pivotal role in memory formation, the presence of the virus in the hippocampal region may modify the host cognitive function. In line with this theory, even thinking about water causes a painful feeling in patients with rabies (Wilson and Rohde, 2019). The rabies virus encodes five proteins. Among them, RVG plays a key role in the pathogenesis of the rabies infection. In the present study, we expressed RVG in the dorsal hippocampus and found that while RVG did not significantly modify spatial acquisition, it strengthens spatial memory performance. This improvement of spatial memory is not attributed to the lentiviral particles as $\triangle \mathrm{RVG}$, like PBS control, had no significant effect on spatial memory task. This effect is probably mediated by its PBM site located at the C-terminus because the PBM-deleted RVG, $\triangle \mathrm{RVG}$, did not change spatial memory formation. Glutamatergic system is particularly important among the diverse molecular signaling pathways and systems involved in spatial memory formation. N-Methyl-d-aspartate (NMDA) and $\alpha$-amino-3-hydroxy-5-methyl-4-isoxazolepropionic acid (AMPA) channels play a pivotal role in modification of synaptic transmission and plasticity in glutamatergic system. Synaptic plasticity is considered a basic mechanism for memory formation. Our results showed that RVG modify the memory through its PBM suggesting that PDZ binding proteins may mediate the boosting effect of RVG on spatial memory. Various PDZ proteins have been identified so far that affect AMPA and NMDA channels and ultimately synaptic transmission and cognition. The well-known PDZ binding protein involved in synaptic neurotransmission is PSD-95, which acts as a scaffold protein to locate NMDA and AMPA receptors in synaptic site (Chen et al, 2015). Other PDZ binding proteins including SAP-97 (Leonard et al, 1998), GRIP/APB (Dong et al, 1997; Srivastava et al, 1998), and PICK1 (Xia et al, 1999) are also reported to interact with AMPA and NMDA receptors to modify synaptic function. It has also been previously reported that the interaction of carboxylic terminal of RVG with PDZ domain of MAST2 and PTPN4 determines cell fate toward survival or apoptosis (Caillet-Saguy et al, 2015; Lafon, 2011). Interestingly, it has been reported that PTPN4 modifies synaptic plasticity in the cerebellum and boosts motor learning by binding to the glutamate receptor type GluD (Kina et al, 2007). Given that PTPN4 is highly expressed in the hippocampus, the effect of RVG on spatial memory may be mediated through 
PTPN4 modification, yet further studies are needed to confirm this assumption and other signaling mechanism(s) engaged in RVG action on cognitive behavior.

Negative emotional memories including fear and anxiety are particularly compromised in rabies infection. Therefore, in this study we also tested impact of RVG on inhibitory avoidance task. We found that along with RVG, $\triangle \mathrm{RVG}$ also reinforces aversive memory. Therefore, it is not possible to establish whether RVG/GFP or $\triangle \mathrm{RVG} / \mathrm{GFP}$ has an effect compared to the PBS control because both lentiviral infection and GFP expression could have effects of their own. We did not test effect of the empty vector that is just expressing GFP in this model of memory. However, it has been shown that intra-hippocampal injection of a lentiviral particles (p156-CMVEGFP, which is a similar construct to ours) with minimum content of transgenes does not change learning and memory of mice in the hippocampal-dependent fear conditioning task (Kunitsyna et $a l, 2016)$. Therefore, it is unlikely that GFP expression per se could improve avoidance memory in our study. Yet, we could not firmly conclude that RVG is able to reinforce aversive memory task in the present study.

Dorsal hippocampus is mainly involved in spatial memory formation and not aversive memory. To be more precise, dorsal hippocampus has pivotal role in spatial memory due to the presence of place cells in this region (O'Keefe and Conway, 1978). On the other hand, amygdala, and bed nucleus of stria terminalis are the main brain regions processing negative emotions (Davis et al, 2010) . These regions have strong innervation to the hippocampus (Goosens, 2011). It is not unlikely that manipulation of hippocampal circuits and corresponding neurotransmission by rabies virus provokes anxiety and aggression. Hippocampus is a large structure having substantial regional differences, each has a distinct gene expression and circuitry (Dong et al, 2009; Lein et al, 2007; Van Strien et al, 2009). While there are robust projections from the neocortex to the dorsal hippocampus, the ventral part has major connection with subcortical regions, including the amygdala. This pattern of circuitry simply leads to the hypothesis that the dorsal hippocampus governs mnemonic learning and the ventral part is involved in emotional processing (Engin et al, 2016; Segal et al, 2010). However, the dorsal hippocampus also regulates emotional and aversive memories through projection to the paraventricular nucleus (Goosens, 2011) indicating the complexity of hippocampal role in negative emotional behavior. Although the ventral hippocampus may play a role in spatial memory, its role in stress and emotional behaviors is much more pronounced. (Lee et al, 2019). Therefore, infecting other regions of the hippocampus, 
including ventral hippocampus, with RVG and assessing the fearful memories and avoidance behaviors will further clarify interplay between rabies virus and cognition-related avoidance behaviors that are seen in rabies patients.

\section{Conclusions}

To our knowledge, this is the first study demonstrating that rabies virus augments hippocampaldependent spatial memory performance through its glycoprotein. Given that RVG mediates binding and fusion of RABV to the cell membrane, it is not unlikely that modification of cell membrane function and subsequent signal transduction are involved in the effect of RVG. The underling molecular and cellular mechanisms need to be disclosed in future studies. On the other hand, both RVG and $\triangle \mathrm{RVG}$ enhanced aversive memory task. In order to certainly adopt a positive role for RVG in aversive memory, effect of the empty lentiviral vector just encoding GFP in dorsal hippocampus should be determined. Given the well-defined role of ventral hippocampus in fear memory, verifying impact of RVG expression in ventral hippocampus on fear memory is suggested.

\section{References}

Bernier BE, Lacagnina AF, Ayoub A, Shue F, Zemelman BV, Krasne FB, Drew MR (2017). Dentate gyrus contributes to retrieval as well as encoding: evidence from context fear conditioning, recall, and extinction. Journal of Neuroscience 37: 6359-6371.

Bouzamondo E, Ladogana A, Tsiang H (1993). Alteration of potassium-evoked 5-HT release from virusinfected rat cortical synaptosomes. Neuroreport 4: 555-558.

Caillet-Saguy C, Maisonneuve P, Delhommel F, Terrien E, Babault N, Lafon M, Cordier F, Wolff N (2015). Strategies to interfere with PDZ-mediated interactions in neurons: what we can learn from the rabies virus. Progress in biophysics and molecular biology 119: 53-59.

Ceccaldi P-E, Fillion M-P, Ermine A, Tsiang H, Fillion G (1993). Rabies virus selectively alters 5-HT1 receptors subtypes in rat brain. European Journal of Pharmacology 245: 129-138.

Chen X, Levy JM, Hou A, Winters C, Azzam R, Sousa AA, Leapman RD, Nicoll RA, Reese TS (2015). PSD-95 family MAGUKs are essential for anchoring AMPA and NMDA receptor complexes at the postsynaptic density. Proceedings of the National Academy of Sciences 112: E6983-E6992.

Davis M, Walker DL, Miles L, Grillon C (2010). Phasic vs sustained fear in rats and humans: role of the extended amygdala in fear vs anxiety. Neuropsychopharmacology 35: 105-135.

Dong H-W, Swanson LW, Chen L, Fanselow MS, Toga AW (2009). Genomic-anatomic evidence for distinct functional domains in hippocampal field CA1. Proceedings of the National Academy of Sciences 106: 11794-11799. 
Dong H, O'Brien RJ, Fung ET, Lanahan AA, Worley PF, Huganir RL (1997). GRIP: a synaptic PDZ domain-containing protein that interacts with AMPA receptors. Nature 386: 279-284.

Dumrongphol H, Srikiatkhachorn A, Hemachudha T, Kotchabhakdi N, Govitrapong P (1996). Alteration of muscarinic acetylcholine receptors in rabies viral-infected dog brains. Journal of the neurological sciences 137: 1-6.

Engin E, Smith KS, Gao Y, Nagy D, Foster RA, Tsvetkov E, Keist R, Crestani F, Fritschy J-M, Bolshakov VY, Hajos M, Heldt SA, Rudolph U (2016). Modulation of anxiety and fear via distinct intrahippocampal circuits. Elife 5: e14120.

Farzaneh M, Sayyah M, Eshraghi HR, Panahi N, Delavar HM, Pourbadie HG (2018). Transduction efficacy and retrograde movement of a lentiviral vector pseudotyped by modified rabies glycoprotein throughout the trisynaptic circuit of the rat hippocampus. The journal of gene medicine 20 :e3046.

Fu ZF, Jackson AC (2005). Neuronal dysfunction and death in rabies virus infection. Journal of neurovirology 11: 101-106.

Ghassemi S, Asgari T,Mirzapour-Delavar H, Aliakbari S, Gholami Pourbadie H, Prehaud C, Lafon M, Gholami A, Azadmanesh K, Naderi N, Sayyah M (2021). Lentiviral Expression of Rabies Virus Glycoprotein in the Rat Hippocampus Strengthens Synaptic Plasticity 1-12.

Goosens KA (2011). Hippocampal regulation of aversive memories. Current opinion in neurobiology 21: 460-466.

Gourmelon P, Briet D, Clarençon D, Court L, Tsiang H (1991). Sleep alterations in experimental street rabies virus infection occur in the absence of major EEG abnormalities. Brain research 554: 159-165.

Hueffer K, Khatri S, Rideout S, Harris MB, Papke RL, Stokes C, Schulte MK (2017). Rabies virus modifies host behaviour through a snake-toxin like region of its glycoprotein that inhibits neurotransmitter receptors in the CNS. Scientific reports 7: 1-8.

Iwata M, Komori S, Unno T, Minamoto N, Ohashi H (1999). Modification of membrane currents in mouse neuroblastoma cells following infection with rabies virus. British journal of pharmacology 126: 1691-1698.

Jackson AC (2016). Diabolical effects of rabies encephalitis. Journal of neurovirology 22: 8-13.

Khan Z, Terrien E, Delhommel F, Lefebvre-Omar C, Bohl D, Vitry S, Bernard C, Ramirez J, Chaffotte A, Ricquier K (2019). Structure-based optimization of a PDZ binding motif within a viral peptide stimulates neurite outgrowth. Journal of biological chemistry 294: 13755-13768.

Kheirbek MA, Drew LJ, Burghardt NS, Costantini DO, Tannenholz L, Ahmari SE, Zeng H, Fenton AA, Hen R (2013). Differential control of learning and anxiety along the dorsoventral axis of the dentate gyrus. Neuron 77: 955-968.

Kina Si, Tezuka T, Kusakawa S, Kishimoto Y, Kakizawa S, Hashimoto K, Ohsugi M, Kiyama Y, Horai R, Sudo K (2007). Involvement of protein-tyrosine phosphatase PTPMEG in motor learning and cerebellar long-term depression. European Journal of Neuroscience 26: 2269-2278. 
Kunitsyna TA, Ivashkina OI, Roshchina MA, Toropova KA, Anokhin KV (2016). Lentiviral transduction of neurons in adult brain: evaluation of inflammatory response and cognitive effects in mice. Bulletin of experimental biology and medicine 161: 316-319.

Ladogana A, Bouzamondo E, Pocchiari M, Tsiang H (1994). Modification of tritiated $\gamma$-amino-n-butyric acid transport in rabies virus-infected primary cortical cultures. Journal of general virology 75: 623-627.

Lafon M (2011). Evasive strategies in rabies virus infection. Advances in virus research 79: 33-53.

Lee SLT, Lew D, Wickenheisser V, Markus E (2019). Interdependence between dorsal and ventral hippocampus during spatial navigation. Brain and behavior 9: e01410.

Lein ES, Hawrylycz MJ, Ao N, Ayres M, Bensinger A, Bernard A, Boe AF, Boguski MS, Brockway KS, Byrnes EJ (2007). Genome-wide atlas of gene expression in the adult mouse brain. Nature 445: 168-176.

Leonard AS, Davare MA, Horne MC, Garner CC, Hell JW (1998). SAP97 is associated with the $\alpha$-amino3-hydroxy-5-methylisoxazole-4-propionic acid receptor GluR1 subunit. Journal of biological chemistry 273: $19518-19524$.

Nourry C, Grant SG, Borg J-PJSS (2003). PDZ domain proteins: plug and play! 2003: re7-re7.

O'Keefe J, Conway D (1978). Hippocampal place units in the freely moving rat: why they fire where they fire. Experimental brain research 31: 573-590.

Paxinos G, Watson C (2006). The rat brain in stereotaxic coordinates: hard cover edition. Elsevier.

Pourbadie HG, Sayyah M, Khoshkholgh-Sima B, Choopani S, Nategh M, Motamedi F, Shokrgozar MA (2018). Early minor stimulation of microglial TLR2 and TLR4 receptors attenuates Alzheimer's diseaserelated cognitive deficit in rats: behavioral, molecular, and electrophysiological evidence. Neurobiology of aging 70: 203-216.

Prehaud C, Lay S, Dietzschold B, Lafon M (2003). Glycoprotein of nonpathogenic rabies viruses is a key determinant of human cell apoptosis. Journal of virology 77: 10537-10547.

Préhaud C, Wolff N, Terrien E, Lafage M, Mégret F, Babault N, Cordier F, Tan GS, Maitrepierre E, Ménager P (2010). Attenuation of rabies virulence: takeover by the cytoplasmic domain of its envelope protein. Science signaling 3: ra5.

Scott CA, Rossiter JP, Andrew RD, Jackson AC (2008). Structural abnormalities in neurons are sufficient to explain the clinical disease and fatal outcome of experimental rabies in yellow fluorescent proteinexpressing transgenic mice. Journal of virology 82: 513-521.

Segal M, Richter-Levin G, Maggio N (2010). Stress-induced dynamic routing of hippocampal connectivity: A hypothesis. Hippocampus 20: 1332-1338.

Song Y, Hou J, Qiao B, Li Y, Xu Y, Duan M, Guan Z, Zhang M, Sun L (2013). Street rabies virus causes dendritic injury and F-actin depolymerization in the hippocampus. Journal of general virology 94: 276-283.

Srivastava S, Osten P, Vilim F, Khatri L, Inman G, States B, Daly C, DeSouza S, Abagyan R, Valtschanoff J (1998). Novel anchorage of GluR2/3 to the postsynaptic density by the AMPA receptor-binding protein ABP. Neuron 21: 581-591. 
Van Strien N, Cappaert N, Witter M (2009). The anatomy of memory: an interactive overview of the parahippocampal-hippocampal network. Nature reviews neuroscience 10: 272-282.

Wilson PJ, Rohde RE (2019). Rabies E-Book: Clinical Considerations and Exposure Evaluations. Dolores Meloni.

Xia J, Zhang X, Staudinger J, Huganir RL (1999). Clustering of AMPA receptors by the synaptic PDZ domain-containing protein PICK1. Neuron 22: 179-187.

Figures and figure captions

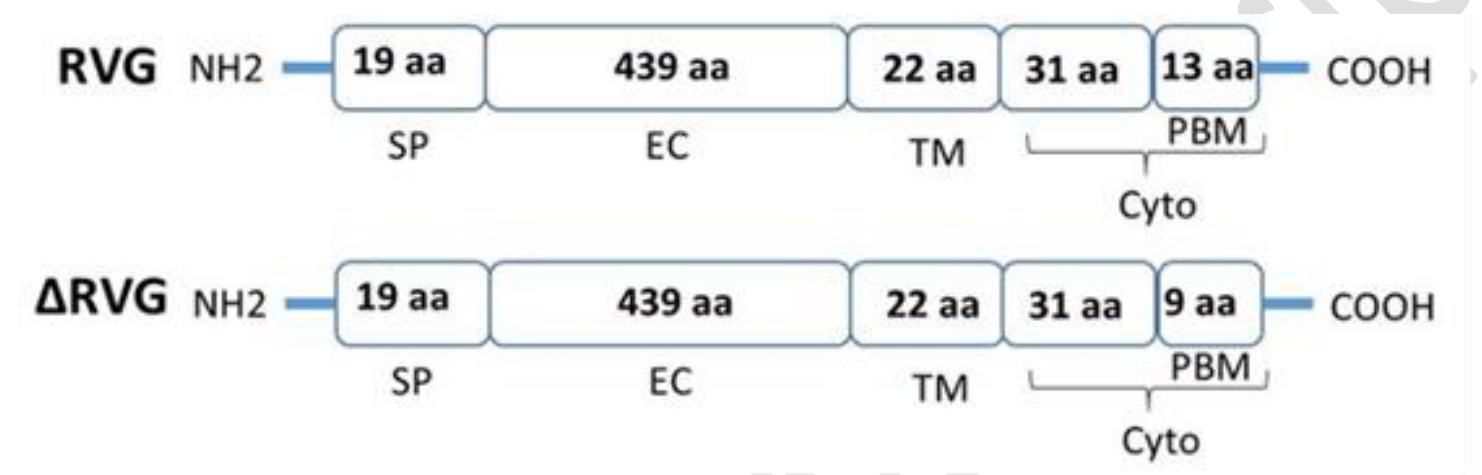

Fig.1. Genomic structure of $R V G$ and $\triangle R V G$. RVG: rabies glycoprotein, $\triangle \mathrm{RVG}$ : rabies glycoprotein with deleted PBM motif. PBM: PDZ binding motif. TM: transmembrane. EC: extracellular. SP: signal peptide. Cyto: cytoplasmic. RVG and $\triangle \mathrm{RVG}$ were subcloned in Plenti7.3 DEST Plasmid containing CMV promoter. 


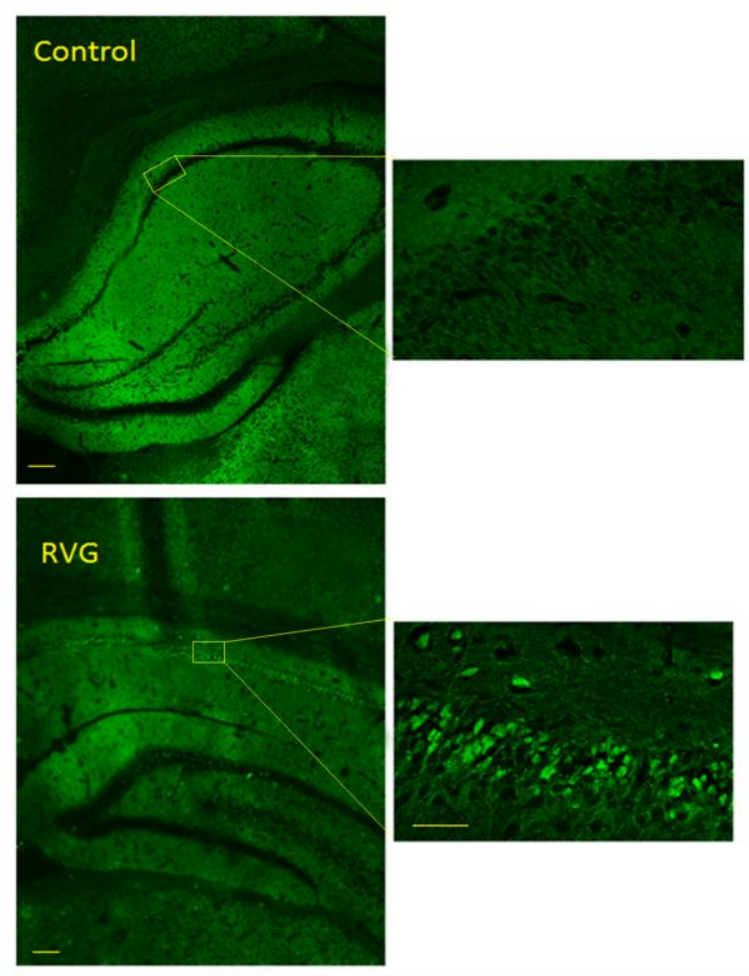

Fig.2. RVG expression in the hippocampal CA1. No fluorescent green cell is observed in the control group. However, in the RVG group, green cells indicate the expression of the RVG/GFP in CA1 pyramidal neurons one week after microinjection of the lentivector into the dorsal hippocampus (magnification: 4 and 20x, scale bar: $50 \mu \mathrm{m}$ ). 
A
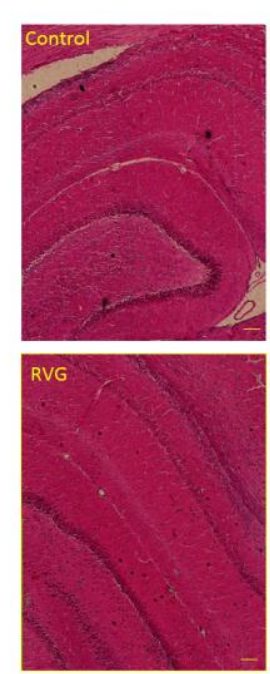

B
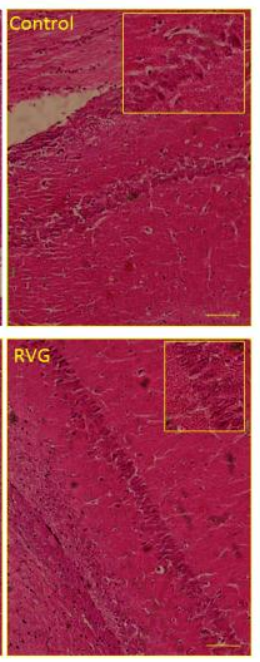

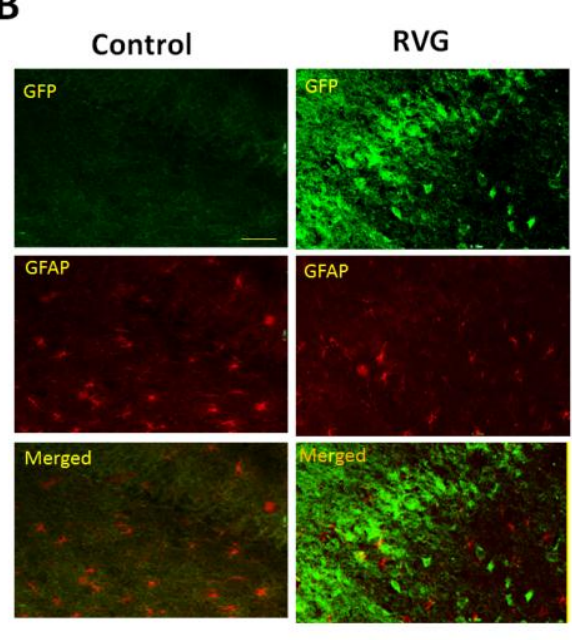

Fig.3. Neuronal and astrocytes morphology one week following RVG expression in the hippocampus. A) H \& E staining of the hippocampus of RVG and control group. Green arrows indicate samples of normal neuronal cells. Scale bar: $100 \mu \mathrm{m}$. B) Immunostaining of glial fibrillary acidic protein (GFAP) in control and RVG groups indicated that RVG expression did not stimulate glial cells in the hippocampal CA1 (injection site) region (magnification: 20x, scale bar: $50 \mu \mathrm{m}$ ). 
A
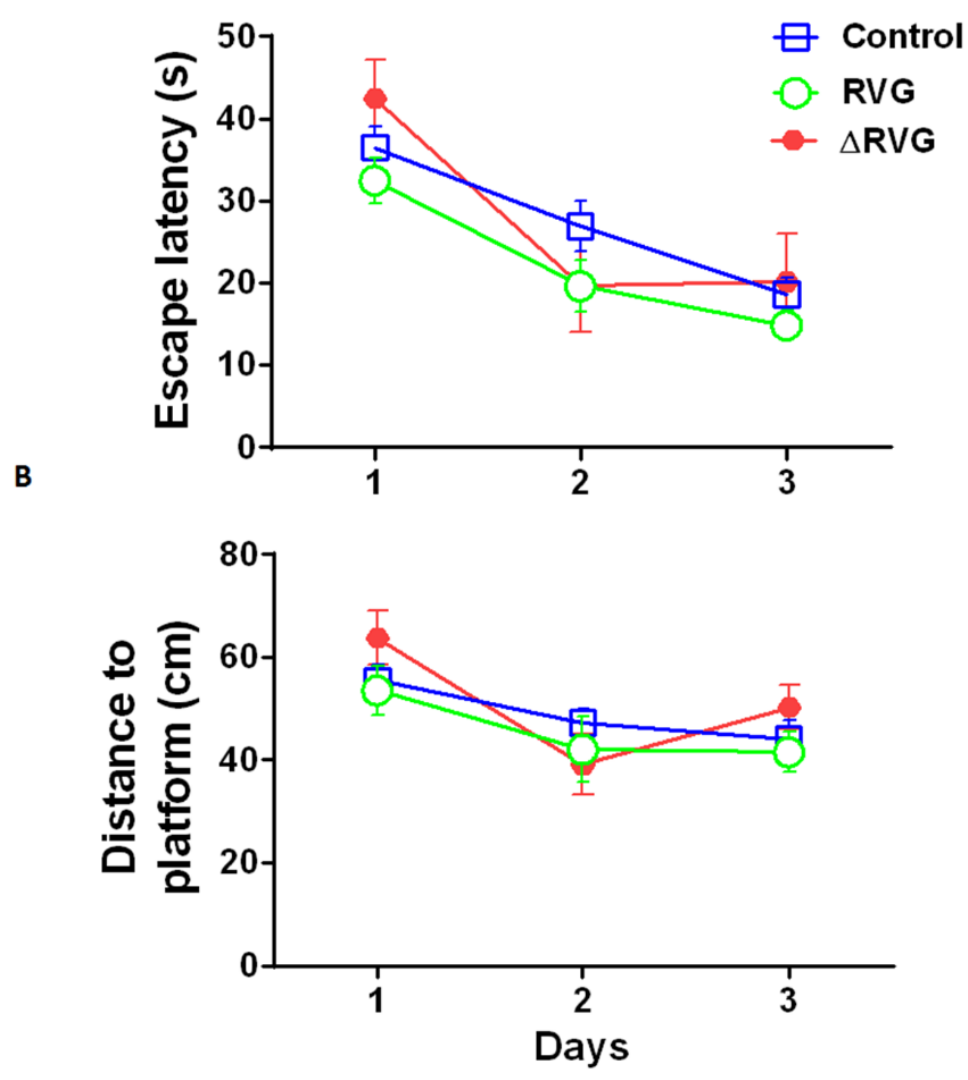

Fig.4. Acquisition of the spatial task in the rats expressing RVG or $\Delta R V G$ in the hippocampal CA1 area. A) No significant difference of spatial acquisition was found among groups. B) No difference of distance swimming from the hidden platform was observed among groups during training days. Data is expressed as Mean \pm SEM. $(n=6-12)$. 
A

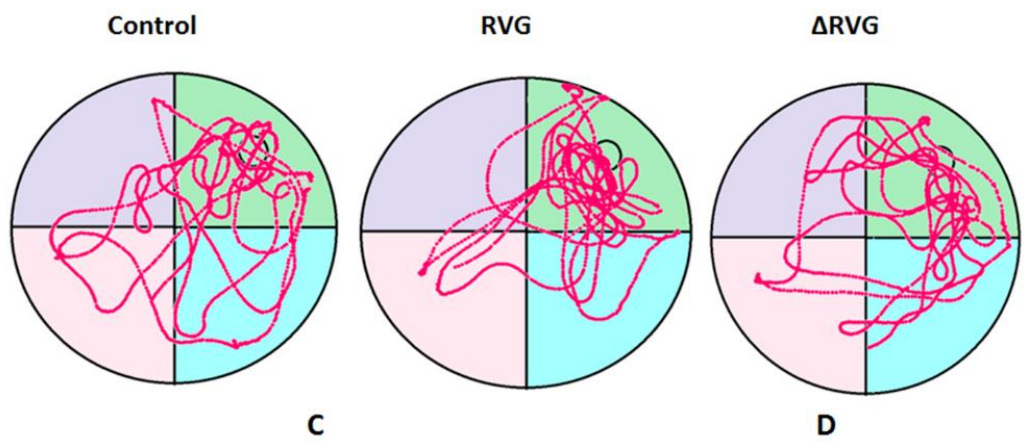

B

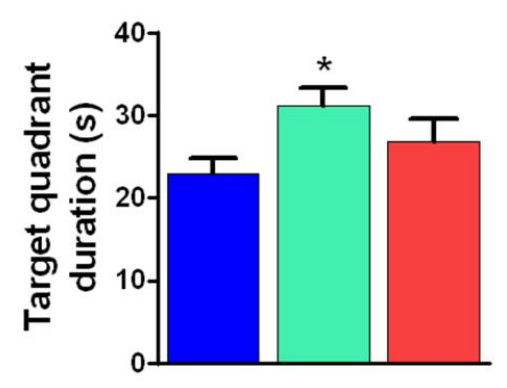

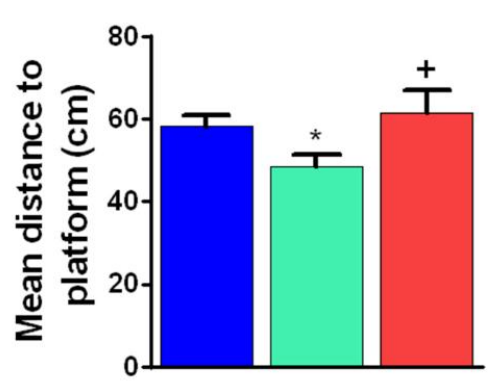

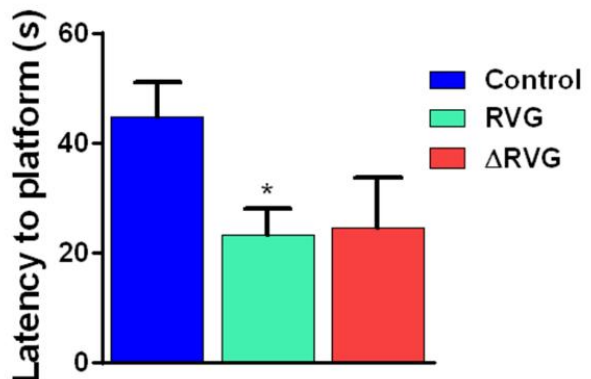

Fig.5. Spatial memory of the rats expressing RVG or $\triangle \mathrm{RVG}$ in the hippocampal CA1 area. A) Representative swim paths during the probe test. B) RVG expression in the CA1 ( $\mathrm{n}=11)$ significantly increased the time spent in the target quadrant compared to the control (PBS-injected) group $(n=10)$. C) The mean distance to the platform significantly decreased in RVG group. In $\Delta R V G$ group $(n=6)$, rats swim significantly farther from the platform location compared to the RVG group. D) RVG expressing rats reach to the platform location faster than control group. No significant difference was observed between control and $\triangle \mathrm{RVG}$ groups in latency to reach the platform position. Data is expressed as Mean \pm SEM. ${ }^{*} p<0.05$ compared to control group. ${ }^{+:} p$ $<0.05$ compared to the RVG group. 

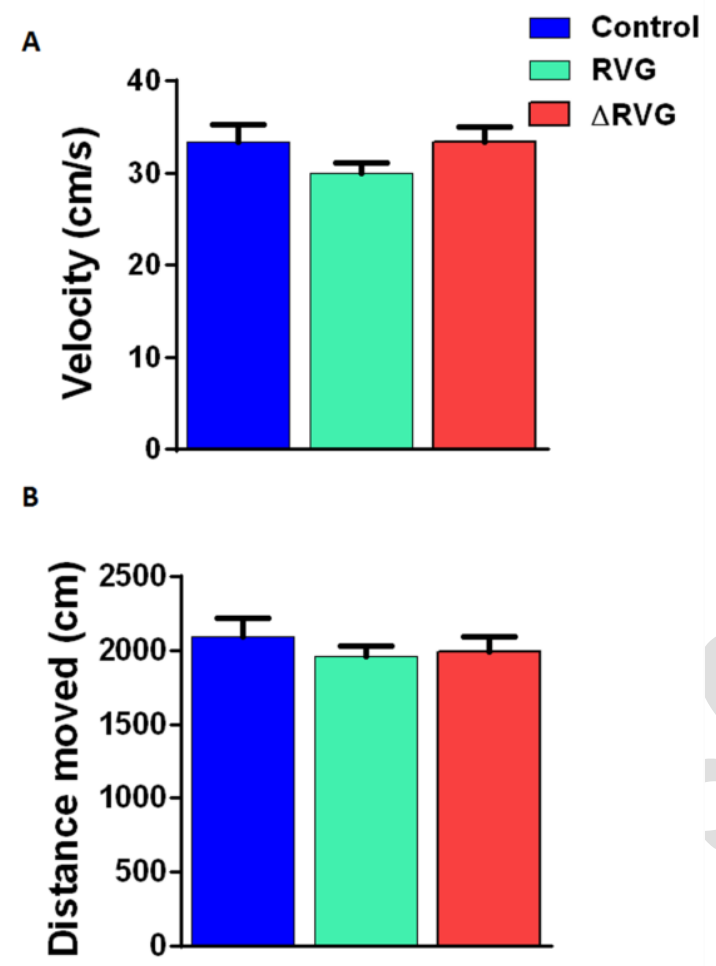

Fig.6. Motor function of rats expressing RVG or $\triangle \mathrm{RVG}$ in the hippocampal CA1 area in the probe test of Morris Water Maze. There was no significant difference in the velocity (A) and the total distance moved (B) among the groups. Histograms represent Mean \pm SEM. 
A

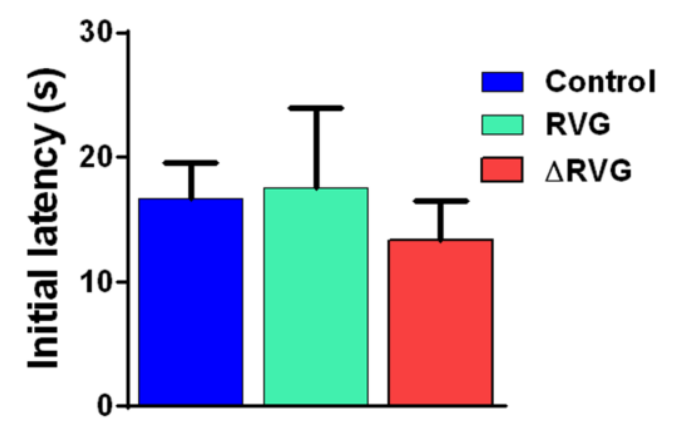

B
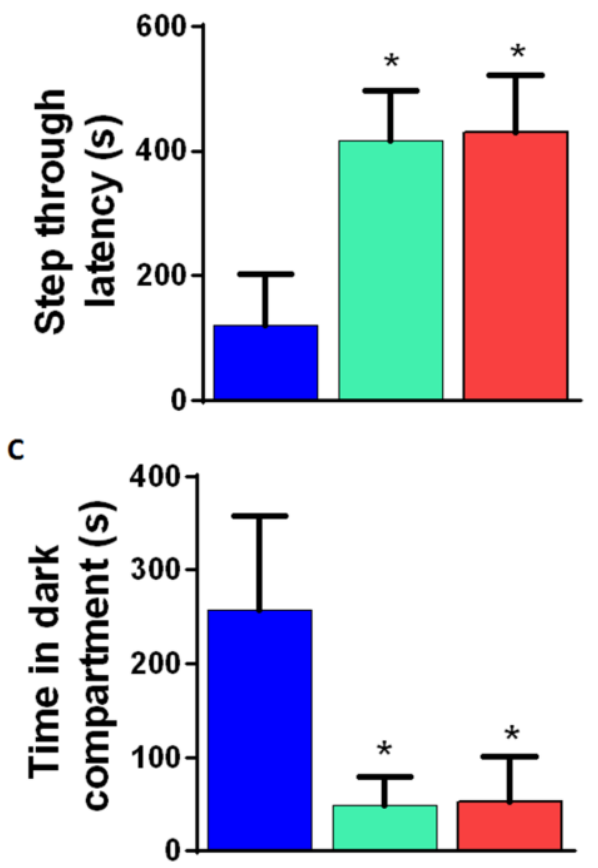

Fig.7. Performance of passive avoidance memory in rats expressing RVG or $\Delta \mathrm{RVG}$ in the hippocampal CA1 area. A) No difference was found between groups in initial latency. B) No significant increase was found in of step through latency between RVG $(p=0.28) / \Delta \mathrm{RVG}(p=$ 0.25) and control (PBS-injected) groups. C) Expression of RVG and $\triangle \mathrm{RVG}$ in the CA1 significantly decreased the time spent in the dark compartment compared to the control group $(p$ $<0.05)$. Data is expressed as mean \pm SEM. ${ }^{*}: p<0.05$ compared to the control group. $(\mathrm{n}=6-7)$.

"This is a pre-print of an article published in J. Neurovirol. The final authenticated version is available online at: https://doi.org/10.1007/s13365-021-00972-2". 